\title{
El proceso de producción de la primera ley argentina de infancia después de la Convención de los Derechos del Niño en Mendoza (1993-1995): la autora, los fundamentos y los magistrados
}

\section{The generation of the first Argentine childhood law following the Convention on the Rights of the Child in Mendoza (1993-1995): the author, the underpinnings and the Magistrates}

\author{
José María Vitaliti ${ }^{1}$ \\ Karen Noel Castillo ${ }^{2}$
}

\begin{abstract}
Resumen
La primera ley de infancia en Argentina después de la Convención de los Derechos del Niño, fue la Ley provincial No 6354 sancionada en 1995. $\mathrm{Si}$ bien se considera un pionero avance en la adecuación jurídica interna, es necesario profundizar en los actores que propusieron y resistieron la sanción de este instrumento jurídico. Este trabajo se propone analizar el proceso de producción de la primera ley argentina de adecuación a la Convención través de la caracterización de la autora-legisladora, los fundamentos de la normativa propuesta y la respuesta del actor judicial, en la primera mitad de la década del 90. La fuente principal utilizada es el proyecto de ley vinculado con fuentes jurídicas e institucionales. Este análisis permitió conocer las resistencias de un poder judicial pragmático y sagaz en su preservación del poder y la astucia de la legisladora en la construcción de estrategias para su sanción.
\end{abstract}

Palabras claves: Mendoza; Minoridad; Derechos del niño.

\begin{abstract}
The first childhood law in Argentina after the Convention on the Rights of the Child was the Provincial Law No. 6354 sanctioned in 1995. Although it is considered a pioneering advance in internal legal adaptation, it is necessary to explore the actors who proposed it and the ones who resisted the sanction of this legal instrument. This paper aims to analyze the production process of the first Argentine law adapted to the Convention through the characterization of the author-legislator, the foundations of the proposed regulations and the response of the judicial actor, in the first half of the 90 decade. The main source used is the drafting legislative, linked to legal and institutional sources. This analysis allowed us to know the resistance of a pragmatic and sagacious judiciary in its preservation of power and the astuteness of the legislator in the construction of strategies for its sanction.
\end{abstract}

Keywords: Mendoza; Minority; Right of the child.

\section{Introducción}

La Ley Provincial No 6354 fue la primera adecuación jurídica a los postulados de la Convención de los Derechos del Niño (en adelante, CDN) en la República Argentina. Fue

\footnotetext{
${ }^{1}$ Lic. en Minoridad y Familia. Doctorando en Ciencias Humanas y Sociales - Universidad Nacional de Quilmes (UNQ). Becario Doctoral INCIHUSA - CONICET. Argentina. Email: jmvitaliti@mendozaconicet.gob.ar; jmvitaliti@gmail.com | https://orcid.org/0000-0003-3404-9753

2 Dra. en Psicología. Becaria doctoral INCIHUSA - CONICET. Argentina. Email: kcastillo@mendozaconicet.gob.ar | https://orcid.org/0000-0003-3517-5452
} 
sancionada por la cámara de Diputados de la provincia de Mendoza en el año 1995, a cinco años de la ratificación de la Convención por medio de la Ley Nacional № 23.849 (1990-1995) y a un año de la inclusión de la Convención en la Constitución Nacional (1994) (LUCERO, 2013). Se popularizó con el adjetivo de "pionera" debido a la osadía de adecuar sustancialmente el sistema jurídico, político e institucional al primer tratado de derechos humanos para las infancias y adolescencias (KONTERLLNIK; MUÑOZ; PELLIZA, 2002, OCAÑA; LAVADO, 2008; FARIAS CARRACEDO, 2014; MOLINA de JUAN, 2017). Tal fue la repercusión de esta normativa que en el Encuentro Federal sobre Políticas de Infancias y Adolescencias realizado en agosto de 1997 en Mendoza, el Director de UNICEF en Argentina, Patricio Fuentes Sarmiento, planteó que:

la Ley 6.354 constituye un hito significativo o hacia la construcción de la ciudadanía de la infancia y de la adolescencia mendocina y un antecedente esperanzado para otros jóvenes argentinos, remarcando el pluralismo político con el cual se llevó adelante esta ley, que representa una adecuación sustantiva a la Convención de los Derechos del Niño. Hoy, esta ley, es el marco con el cual se pretende llegar al Congreso de la Nación, para transformarla en ley nacional (CONSEJO FEDERAL DE DESARROLLO SOCIAL; SECRETARÍA DE DESARROLLO SOCIAL DE LA PRESIDENCIA DE LA NACIÓN; ASOCIACIÓN DE MAGISTRADOS Y FUNCIONARIOS DE LA JUSTICIA DE MENORES; SECRETARIADO NACIONAL PARA LA FAMILIA DE LA CONFERENCIA EPISCOPAL ARGENTINA; UNICEF, 1998, PODER LEGISLATIVO DE LA PROVINCIA DE TIERRA DEL FUEGO, 1997).

La convención es el nuevo marco mundial de respeto por los derechos de niños/as y adolescente. Su contenido posee carácter instructivo, es decir, no vale como ley, pero sirve como parámetro para las legislaciones de los países ratificantes del tratado. Representa, a su vez, un momento de inflexión, ya que es un punto de llegada de debates en instancias internacionales que ya se venían realizando como: la Declaración de los derechos del niño en 1959, la designación en 1979 del Año Internacional del Niño, las Reglas mínimas de las Naciones Unidas para la administración de la justicia de menores "Reglas de Beijing" en 1985; $\mathrm{y}$, un punto de partida, ya que se trata del paradigma, debido a las transformaciones que supone.

A nivel latinoamericano, Beloff (1999) identifica las trasformaciones jurídicas latinoamericanas surgidas entre 1900-2000 en tres grupos. En primer lugar, aquellos países en que la ratificación de la CDN no tuvo ningún impacto o tuvo un impacto político superficial o retórico. El segundo, los países que llevaron a cabo un proceso de adecuación formal o eufemística de las leyes internas del país a la CDN. Por último, el tercer grupo, los países que realizaron una adecuación sustancial de las leyes internas del país a la CDN. Argentina se situaría en el segundo grupo. 
De esta manera, el análisis latinoamericano propuesto por Beloff (1999) plantea en lo Nacional una adecuación superficial, mientras que traslocando el análisis hacia el territorio nacional podríamos afirmar que la ley provincial ya mencionada inaugura el proceso de modificación de la legislación en otras provincias de Argentina. Estas diferencias entre lo superficial y sustancial constituyeron un verdadero obstáculo para las regulaciones provinciales, ya que continuaba vigente en "lo Nacional", la ley de Patronato de Menores $\mathrm{N}^{\circ}$ 10.903 y posteriores normativas surgidas en gobiernos dictatoriales que regulaban la situación de niños/as y adolescentes.

El marco que contiene este análisis se delimita en torno a la división de dos paradigmas. El paradigma de la Situación Irregular y el de la protección integral, cuyo punto de inflexión es la promulgación de la CDN. El paradigma de Situación Irregular, o también llamado "modelo tutelar", "filantrópico", o "asistencialista"; está integrado por discursos, prácticas, instituciones y leyes [que] respondían a un esquema que tenía la consideración del menor como objeto de protección, circunstancia que legitimaba prácticas peno-custodiales y represivas encubiertas (BELOFF, 1999). La misma autora, plantea que a partir de la CDN se comienza a debatir las maneras de interpretar la relación con la infancia, tradicionalmente encarada desde esa perspectiva asistencialista y tutelar, que cedió frente a un planteo de la cuestión en términos de ciudadanía y de derechos para los más jóvenes.

En oposición al anterior, el Paradigma de la Protección Integral concibe a los/as niños/as como sujeto de derechos-ciudadanos, y por lo tanto, deben formularse políticas públicas que atiendan a la etapa del desarrollo en que se encuentra. Si bien el Estado es el garante de las acciones para/con la infancia, la responsabilidad por las acciones es compartida por la familia y la comunidad (GOMES DA COSTA, 1993; PETRY VERONESE, 2017). En la Argentina, este paradigma comienza con la ratificación de la CDN que la incorporó inmediatamente al plexo legal a través de la Ley Nacional $N^{\circ} 23.849 / 90$ y a la Constitución Nacional en el año 1994. Once años más tarde, se sanciona la Ley 26.061/2005 de Protección Integral de los Derechos de niños/as y adolescentes, que crea instituciones, autoridades, medidas y principios que ofrezcan mayores garantías de aplicación de los Derechos del niño en el territorio nacional.

Estos paradigmas se plantearon como discursos antagónicos a partir de la CDN. Se generó un "escenario dicotomizado por lo viejo-malo a destituir y lo nuevo-bueno a instaurar" (LLOBET, 2013, p. 217). A su vez, la ansiedad de cambio de diferentes sectores implicó la instalación de categorías clasificatorias para uno y otro paradigma, estableciendo un intento previo y una vara provisoria para medir nuevas maneras de hablar y actuar en la protección de 
las infancias. Sin embargo, esta idealización discursiva colocó en un ideal superador a los nuevos paradigmas, con el riesgo de esencializar los derechos configurándolos como un ente reificado y etéreo, ajeno a las prácticas sociales e históricas (BARNA, 2012). Ante este riesgo esencializante, es menester situar la producción histórica y social en base a develar las configuraciones y tensiones que produjeron la emergencia de los productos jurídicos, a partir de las tensiones, estrategias y limitaciones histórico-sociales de los actores intervinientes y los discursos vigentes.

De esta manera, la presentación de la ley provincial de Mendoza $^{1}$ como instrumento jurídico local con ansias de cambio en lo nacional, ha sido estudiada por un acotado grupo de autores/as que han contribuido con sustento sus miradas. Las argumentaciones planteadas difieren entre aquellas donde la ley es un avance para la época (KONTERLLNIK; MUÑOZ; PELLIZA, 2002; OCAÑA; LAVADO, 2008; FARIAS CARRACEDO, 2014; MOLINA de JUAN, 2017),y aquellas donde existe la falta de despegue de la tutelaridad de las medidas judiciales (LUCERO, 2013) y los dilemas de implementación y la continuidad de prácticas perteneciente a la Doctrina de la Situación Irregular (OCAÑA, 2007; OCAÑA; LAVADO, 2008; FARIAS CARRACEDO, 2014). Sin embargo, dichas miradas no han planteado quienes fueron los autores/as, los motivos o fundamentos de quienes presentaron el proyecto de ley y los actores que tensionaron y resistieron la nueva propuesta, que para este caso será el actor judicial.

Quien introduce una mirada somera sobre el actor judicial en la producción de la ley, ha sido en Ocaña y Lavado (2008) quienes plantean que "las resistencias se referían al cambio en los roles de los magistrados, sobre todo por la larga vigencia de la Doctrina de la Situación Irregular $^{2}$ plasmada en la antigua ley en la que confiaban ciegamente" (p. 391). Sin embargo, no profundiza en cuáles fueron estas resistencias en el proceso de producción de la ley.

La importancia de indagar lo antes dicho radica en comprender la configuración del poder judicial, en especial la justicia de familia, como un actor que ha tensionado las definiciones y ha conflictuado competencias en torno al vínculo entre éste y el poder administrador en relación con la cuestión de la niñez y adolescencia.

A partir de lo antes dicho, el propósito del presente trabajo es analizar el proceso de producción de la primera ley argentina de adecuación a la CDN a través de la caracterización de la autora-legisladora, los fundamentos de la normativa propuesta y la respuesta del actor judicial, en la primera mitad de la década del 90. La fuente principal utilizada será el proyecto de ley de la Ley Provincial No 6354, que contiene 347 fojas y está compuesto por documentos 
diversos como: los fundamentos y el primer borrador de la ley, diferentes proyectos de ley con modificaciones, escritos que actúan de canalizadores del actuar legislativo, resoluciones de cámaras y comisiones y, por último, documentos relacionados durante el proceso legislativo y posteriores a la promulgación. Asimismo, se facilitó a partir de la indagación en el Archivo Legislativo de la provincia de Mendoza.

En primer lugar, presentaremos a la autora de la ley y el ámbito desde el cual defendió sus propuestas. En segundo lugar, realizaremos un análisis de los fundamentos del proyecto de ley, describiendo los hitos o inicios de un discurso que después sería fuertemente dicotomizado e idealizado. En tercer lugar, profundizaremos en la postura de los magistrados, quienes se manifiestan mediante una carta enviada a la cámara de senadores de la provincia de Mendoza, estableciendo adhesiones parciales y pragmáticas y resistencias totalizantes de la nueva propuesta.

\section{Las legisladoras de "la cresta de la ola"3 y la composición patriarcal de la cámara}

El proyecto de ley fue presentado el 29 de septiembre de 1993 en la Honorable Cámara de Diputados, por la senadora provincial María Teresa Oldrá de Berchessi (FREJUPO) ${ }^{4}$ en coautoría con los diputados Ángel José Santolín (Alianza de Centro) ${ }^{5}$ y Dante Arturo Zancan (U.C.R. $)^{6}$. La autora con mayor referencia fue la primera y su estrategia política para legitimar su aceptación como propuesta fue la trasversalidad partidaria. Sin embargo, no fue la única estrategia.

La senadora María Teresa Oldrá de Berchessi fue Interventora de la Dirección Provincial del Menor en 1987 (DECRETO N 5260/1987) hasta su renuncia en el año 1990 (DECRETO No 817/1990). Su impronta de gestión se caracterizó por la implementación de programas específicos como: programa preventivo, programa de guarderías, jardines maternales, comedores y centros preventivos y asistenciales, programa de prevención de la violencia doméstica y asistencia a la mujer en situación de mal trato, programa de asistencia al niño en su hogar, programa de amas externas, programa familia cuidadora, programa de chicos de la calle y programa de menores en situación de riesgo y/o infractores ilegales correccionales (OLDRÁ DE BERCHESSI, 1990). La iniciación de la política de desmasificación de los macrohogares ${ }^{7} \mathrm{o}$ macroinstituciones de internación, también comenzó en este periodo (OLDRÁ DE BERCHESSI, 1990; FARIAS CARRACEDO, 2014), propiciando la creación y renominación de los pequeños hogares en minihogares. Esta política consistía en la reducción de grupos de convivencia, la coordinación con la política de vivienda para dotar a "familias 
carentes de este bien", y la creación de minihogares que evitaran el desarraigo de los niños (OLDRÁ DE BERCHESSI, 1990). Estas acciones planificadas cristalizaban una "reivindicación específica y fuerte" que las mujeres plantearon en el Encuentro Nacional de Mujeres organizado en Mendoza en el año 1988 (ALVARADO; BARÓN DEL PÓPOLO, 2019).

En 1989 asume como diputada provincial por la tercera sección electoral en el Frente Nacional FREJUPO. Fue reelecta en el año 1993 aunque como senadora provincial por la misma sección electoral en el Partido Justicialista. Fue autora y coautora de 13 proyectos de ley, de los cuales el $60 \%$ fueron leyes provinciales. En muchos de sus proyectos de ley, tuvo contacto en la gestión o a través de luchas sociales. Su participación en la comisión organizadora del Tercer Encuentro Nacional de Mujeres (ALVARADO; BARÓN DEL PÓPOLO, 2019) realizado en Mendoza en el año 1988 y luego redactando junto a Alberto Montbrun (UCR) permitió presentar la ley que modificó el régimen electoral en lo referente al cupo femenino $\mathrm{N}^{\mathrm{o}}$ 5888/1992 (CAMINOTTI, 2008). Lo mismo sucedió con la Ley provincial $\mathrm{N}^{\mathrm{o}}$ 6354, ya que como expresamos en lo anterior, fue Interventora de la Dirección Provincial del Menor.

A partir de la vuelta de la democracia (1983), las mujeres comenzaron a integrar el poder legislativo y, por lo tanto, las listas de postulaciones de los partidos políticos, representaban un porcentaje menor al $15 \%$ del total masculino de legisladores ${ }^{8}(13 \%$ en Senadores y $15 \%$ en Diputados). El clima de época se caracterizaba por improntas patriarcales, y, en consecuencia, temas impulsados por las mujeres eran deslegitimado y encorsetados a su función en la familia tradicional $^{9}$.

En principio, esta composición, vislumbraba una predominancia del partido de la legisladora autora de la Ley $N^{\circ} 6354$ augurando una legitimación de la propuesta. Sin embargo, la deslegitimación de los temas traídos por las mujeres legisladores y los intereses en una legislación de infancia que podrían representar un conflicto de poderes afectando potestades existentes hasta el momento, dilatarían el tratamiento de este proyecto de ley, como veremos más adelante.

\section{Denominación y fundamentos del nuevo proyecto}

El proyecto de ley ${ }^{10}$, en primera instancia se denominó de la siguiente manera: "Creando en el ámbito de la gobernación el Consejo Provincial del niño y el adolescente”. Luego en el mismo expediente se anuló y se instaló la nueva denominación: "Estableciendo la protección 
del niño y el adolescente". La anulación primera sugiere que el proyecto pretendería focalizarse en un dispositivo posible donde se afectara a personal dentro del poder ejecutivo. Este dispositivo buscaba motorizar aquellos cambios esperados en justicia, políticas y descentralización de recursos. Esta propuesta ya se encontraba vigente, por ejemplo, en la provincia de Misiones Decreto No 1104/1990 y en la provincia de Tierra del Fuego mediante la ley $N^{o}$ 20/1992. Era un dispositivo conocido en la época, por ello, su aprobación implicaría una resolución menos dificultosa que cambiar la estructura completa de protección de la infancia. Por otro lado, en el recorrido que realizaremos en lo siguiente, los fundamentos del proyecto de ley inclinan la balanza hacia una mayor justificación del "Consejo". No obstante, el cambio de denominación implicó más que un reemplazo del titular, sino la reconfiguración estructural del sistema sobre el que se basa la protección de los niños/as y adolescentes. Esto se debió a la influencia de un organismo internacional a través Emilio García Méndez consultor para Latinoamérica y Caribe de $\mathrm{UNICEF}^{11}$ quien facilitó el nuevo modelo de proyecto de ley, con adecuaciones del consultor y de la autora de la ley.

Luego de la denominación, se plantearon los fundamentos o razones que legitiman y justifican la sanción de la norma (PINEDA NAVAS, 2001). En primer lugar, es importante destacar que el proyecto de ley se presentó un año antes de la Constitución Nacional realizadas en 1994. Si bien existía una ley que ratifica la Convención aun no tenía jerarquía constitucional y esto se vio en los antecedentes jurídicos planteados en los fundamentos. En segundo lugar, se propone una relectura del pasado a través del análisis de ley $\mathrm{N}^{\circ} 1304$. En tercer lugar, se propicia una crítica desde un discurso en formación o prediscurso, que luego sería dicotomizante/dicotomizado entre el nuevo y viejo paradigma (LLOBET, 2013; BARNA, 2012). Este prediscurso motoriza dos planteos: las legislaciones de minoridad sostenidas en la Doctrina de la Situación Irregular y los postulados de la CDN postulados en este nuevo encuadre para las nuevas legislaciones. Ambos planteos se abordan como críticas teóricas hacia lo existente: una realidad legitimada por el poder real y que cuenta 54 años de ejercicio (ley 1304). Para finalizar se expone los puntos principales de la nueva propuesta.

A continuación, se plantean los fundamentos que acompañaron a este proyecto de ley que luego, mediante diferentes tratamientos, se convertirían en el primer ensayo jurídico provincial y serviría como mojón de - o impulso para - la adecuación para el terreno nacional. Primer fundamento: La ley 1304 como el problema "realmente existente"

El primer fundamento sobre el que se posicionan para el cambio de lo existente, comienza con la descripción y análisis de la normativa existente: la Ley provincial $\mathrm{N}^{\circ}$ 
1304/1939 Patronato y Tribunales de Menores. Según la posición de los/as legisladores la ley emerge apuntalada por los problemas sociales existentes en la época de surgimiento: "huérfanos producto de las familias que desaparecían por enfermedades infecto contagiosas y otras muertes" (PROYECTO DE LEY No 6354, p. 3). Una de las enfermedades infecto-contagiosas que invadió a la Argentina es la gripe española (1918-1919) que afectó con mayor incidencia de muertes a la región norte y cuyo, y en los sectores más pobres (CARBONETTI, 2010). Carbonetti, destaca que la epidemia reveló un problema mayor:

la epidemia de gripe, por su dimensión y por su impacto, desnudó las falencias de un Estado que todavía estaba en conformación, la impotencia de una medicina que aún no se consolidaba como garante de la salud y el desequilibrio económico y geográfico de la sociedad (CARBONETTI, 2010, p. 174).

En la perspectiva de los/as legisladores/as de la nueva propuesta de ley, la orfandad como categoría histórica, sirvió como motor del surgimiento de la ley № 1304 y se apoyó en la “prevención de menores en situación de riesgo, abandono moral o material a través de la política de minoridad" (PROYECTO DE LEY Nº 6354, p. 3) como alternativa de respuesta al fenómeno social. Con este fin, se crea un organismo tutelar, organizado por el fuero judicial, cuya función primordial era:

intervenir en la ayuda a menores cuya precaria situación económica, los pusiera en peligro o en condiciones perjudiciales para su salud, para su moral o para su educación o instrucción, además de la protección de huérfanos, desvalidos o abandonados, moral o materialmente y de los que acusaren perversión o peligrosidad, así como los que hubieran sido víctimas de infracciones o delitos o los que lo hubieran cometido (PROYECTO DE LEY N 6354, p. 3).

$\mathrm{Al}$ analizar el problema social que plantearon los/as legisladores/as como el surgimiento de la ley de patronato, excedería las funciones del organismo tutelar anteriormente citado. Esta macrofunción adjudicada al organismo, no legisla únicamente sobre huérfanos, sino, además, sobre la situación precaria de los niños/as o adolescentes, que se denomina como: perversión o peligrosidad y delincuencia.

El actor mediante el cual la ley 1304 se ejecuta es principalmente el poder judicial, creado para atender "desde los riesgos sociales a los problemas civiles y penales" (PROYECTO DE LEY $\mathrm{N}^{\circ}$ 6354, p. 3). El procedimiento judicial incluía la interrogación directa de los/as implicados/as en la acción judicial y la incorporación en la ley del juicio oral. Otro de los actores que juegan en esta política sobre la minoridad se crean mediante el fomento de la 
corresponsabilidad a través de la participación de instituciones privadas en la contención de “menores en riesgo" (PROYECTO DE LEY No 6354, p. 3).

Este rescate de los elementos principales de la ley local en la primera parte de los fundamentos del proyecto de ley 6354, pretenden presentar una descripción sobre los aspectos fundamentales y el contexto de emergencia de la ley local. Sin embargo, este rescate carece de aspectos significativos del contexto, como por ejemplo, que las leyes de control sobre ciertos sectores de la población surgen por la "efervescente protesta social" (BRACHETTA; BRAGONI; MELLADO; PELAGATTI, 2019), donde la regulación de las infancias resultaba prioritario debido a la concepción de peligrosidad y delito vigente hasta el momento y a la participación de los niños/as y adolescentes en las manifestaciones sociales junto a sus familiares (ZAPIOLA, 2010).

Por otro lado, en toda producción de la normatividad, opera la fuerza propia de su configuración - vis formae - que implican un proceso de descontextualización y de desconexión argumentativa entre el problema social y la respuesta al mismo (CASAS, 2006), propia de la invisibilización del control social y la violencia legitimada del Estado (BOURDIEU, 1986). Asimismo, la utopía de cambio del/a legislador/a cabalga junto a la miopía propia de la época, en la cual se destaca la voluntad por modificar la ley local con 54 años de vigencia frente a los recientes 4 años de la ratificación de la $\mathrm{CDN}$.

Otro de los fundamentos planteados, opera una colocación de la ley $\mathrm{N}^{\mathrm{o}} 1304$ en la trayectoria de las "legislaciones de minoridad en riesgo" (PROYECTO DE LEY No 6354, p. 3) donde no hay distinción entre abandonado y delincuencia. Esta trayectoria legislativa se caracteriza por la judicialización del problema de la minoridad donde la norma jurídica reemplaza la política pública. De esta forma, según el proyecto de ley, la escasez de recursos en los mismos sectores sociales facilitó el manejo jurídico del problema social.

Continuando con lo anterior, esta incrustación de la ley local en las legislaciones minoristas, es un aspecto negativo, ya que se configura al sujeto como peligroso a partir de las teorías positivistas ${ }^{12}$ en las que se basa el derecho de menores. Las claves de esta configuración se pueden considerar a partir de: la vinculación de peligrosidad con el delito; la falta de discriminación entre pobreza y la inmoralidad; la extensión de concepto de delincuencia a sectores marginados, y la perversión ${ }^{13}$ que alcanzaba a todos por igual, infringiera o no a la ley. Incluyendo aquellos que eran inadaptados en mayor o menor grado, y que no habían infringido la ley, como pobres, analfabetos, anormales, deficientes y predispuestos al delito (PROYECTO DE LEY No 6354). 
Más allá de lo explicitado en el proyecto de ley, las legislaciones de menores en riesgo cristalizaban la configuración de la peligrosidad como una consecuencia posible en aquellos/as consideradas pobres o desamparados. Según Robert Castel (1986) la construcción de la peligrosidad en el proletariado, es posterior a peligrosidad per se. Esto se debe a que la peligrosidad se presenta como concepto ambiguo y dificultoso, determinado en una doble vía: 1- es una categoría inmanente u ontológica, se es peligroso y 2- la evidencia o prueba de peligro se recoge cuando el hecho se haya consumado, es decir a des-tiempo; solo existen imputaciones o hipótesis de peligrosidad. Bajo este régimen categorial, todos/as son portadores de una amenaza o son peligrosos y no se puede encerrar a todos los que se sospecha de peligroso. De esta manera, peligrosidad en las capas desfavorecidas se consolida como representación social.

A partir de ello, el encierro o la internación es la tecnología preventiva privilegiada junto con esterilizar. Encerrar es neutralizar, si es posible por adelantado al sujeto supuestamente peligroso (CASTEL, 1986). El individuo es trasladado a un nuevo medio para que se vea impedido de desarrollar la amenaza de la que es portador. Mientras que, la esterilización como práctica eugenésica (principio de preservación de la raza) con el fin no de tratar al individuo en particular sino de procurar que no transmita la amenaza de la que es portador.

A su vez, el concepto de peligro transciende hacia lo causativo por medio del riesgo. El riesgo material o moral como posibilidad de educación correctiva significaría una respuesta social al problema de la pobreza (CERDÁ, 2013). De esta manera, riesgo y peligrosidad se fusionan para actuar como tecnología de control de las poblaciones más desfavorecidas.

\section{Crítica teórica de lo real I: la Doctrina de la Situación Irregular}

La retórica sobre los paradigmas estuvo presente en los fundamentos de este proyecto de ley a través de la doctrina de situación irregular. Se lo califica como una "concepción teórica" que ha servido para colocar a la minoridad como un "problema marginal" en lugar de ser jerarquizado como "problema social”. Esta marginalidad de la minoridad recorta las ventajas que pueden acontecer al ser priorizadas por las políticas sociales estatales. En definitiva, la minoridad no es problema legitimado por el Estado "administrador" y sus políticas sociales, ya que donde no hay un problema social, las respuestas político-sociales no tiene gran calibre. No obstante, esta idea primaria invisibiliza al Estado como "juzgador" que ha tenido un papel predominante en los temas sobre la minoridad (PROYECTO DE LEY No 6354, p. 4).

Se expresa, además, que la minoridad ha sido estigmatizada a través del constructo: "menor delincuente abandonado". El Estado "benefactor" atenúa situaciones emergentes de sectores sociales más carenciados, en las que se encuentran estas minoridades estigmatizadas 
(PROYECTO DE LEY N ${ }^{\circ}$ 6354). Esta situación es anunciada y denunciada como tratado discriminatorio, funcionando como una lógica cíclica de reproducción.

A su vez, el/la redactor/a legislativo/a entiende que en la Doctrina de la Situación Irregular el constructo "Pobre-abandonado-delincuente" es un axioma. La definición de axioma indica que: pobre, abandonado y delincuente, son situaciones sociales y subjetivas que se enmarcan en una criminalización o en un enmarcado cuyo objetivo es la erradicación del delito.

Para afirmar este argumento, donde la pobreza está criminalizada, el/la redactora legislativa recurren a diversos criterios. En primer lugar, expresan que:

la delincuencia no es exclusiva de ciertos sectores sociales, pertenece a todos los estratos que componen la sociedad, hoy tal vez en germen, en amplios sectores de las clases altas, donde del abandono moral y espiritual conlleva a grandes insatisfacciones, que generan hechos delictivos y conductas antisociales (PROYECTO DE LEY N ${ }^{\circ}$ 6354, p. 4).

En las palabras en negritas observamos que la delincuencia es sospechada en otros sectores sociales altos como germen, y que tanto hechos delictivos como conductas antisociales se producirían por abandonos que no son materiales, sino que podrían devenir de la carencia moral o espiritual. A partir de este primer criterio nos preguntamos: ¿Qué tipo de delincuencia es la que significan? ¿Cuáles son los actos delictivos que quedarían por fuera de esta segregación de delitos de la pobreza? ¿Por qué en los “sectores de clase alta” la delincuencia se sospecha, mientras que en "la pobreza" es difícil desasir el vínculo con la delincuencia? Al parecer, esta debilidad en el argumento que pretende problematizar los postulados de la Doctrina de la Situación Irregular, recursivamente intenta resolver un postulado mediante un prejuicio.

El segundo criterio referencia elementos propios del etiquetamiento social presentes en la criminalización de ciertos perfiles. Se plantea que:

insistir en el enlace pobre-abandonado-delincuente consolida un perfil de adolescentes infractor que pasa por los Tribunales de Menores, que prematuramente ingresan a sistemas antisociales con códigos propios que les proporcionan identidad. Así su conducta continuará siendo criminalizada en alto porcentaje de llegar a la mayoría de edad (PROYECTO DE LEY No 6354, p. 4).

Se observa la configuración de un perfil producto de una identidad en la que el constructo pobreza, abandono y delincuencia están galvanizados y comprometidos como criterios de selección, clasificación y constitución de ciertas subjetividades con ciertas características más propensas a la aplicación de la ley que otras subjetividades con otras características. Esta perspectiva donde la criminalización está ligada a la estigmatización, tiene 
su base en la constitución del delito a través de la interacción entre un grupo dominante y otro marginal.

El grupo más fuerte logra definir comportamientos ilegales contrarios a sus propios intereses. El poder de definición y persecución de los propios intereses y fines es la crítica que hace Alessandro Baratta cuando manifiesta que se busca la protección de los sectores poderosos al incluir a los sectores marginales solo para disminuir la afectación de los intereses de los sectores privilegiados (SALINAS, 2020, p. 42).

Es por ello que el tercer criterio plantea que: "el no estar incluidos adecuadamente hasta que una franja de estos menores quede expulsada y no estén contemplados en un sistema no estigmatizado [...]" (PROYECTO DE LEY Nº 6354, p. 5). Apunta, además, a que este proceso de etiquetamiento social tiene la grave consecuencia del encierro "La mayoría [...] pasan gran parte de su juventud en institución de rehabilitación" (PROYECTO DE LEY No 6354, p. 5).

Por otra parte, afirman que la expulsión de estos "menores" producto del etiquetamiento social no estarán contenidos "para su correcta socialización” (PROYECTO DE LEY N 6354, p. 5). Si bien, en lo precedente, se posiciona críticamente entendiendo el derecho penal operando selectivamente, en esta oportunidad reposiciona su argumento hacia las teorías de la subcultura criminal al decir que la contención sería por medio de la resocialización, es decir desaprendiendo "de los comportamientos desviados en los grupos marginados y en las bandas juveniles" (SALINAS, 2020, p. 31). Asimismo, podríamos decir que representa un fundamento propio de su época, ya que es García Méndez quien no critica la re-socialización sino que crítica:

La miseria de los programas de re socialización el tratamiento indiferenciado de menores supuestamente abandonados y supuestamente delincuentes, y los miles de jóvenes confinados en instituciones penitenciarias para adultos, constituyen sólo la punta del iceberg de un inmenso proceso de mistificación (GARCIA MÉNDEZ, 1995, p. 8).

Por último, como parte de la Doctrina de la Situación Irregular, se produce una crítica hacia el Estado y sus medidas de carácter asistencialista. Se afirma que:

Las medidas asistencialistas, por su característica de ser acciones coyunturales, empobrecen el rol fundamental del Estado, porque deslucen la ejecución de políticas a largo plazo que permitan optimizar el futuro de los niños y adolescentes (PROYECTO DE LEY No 6354, p. 5).

A su vez, construyen en su retórica dos constructos: "menor-abandonado-delincuente" y "pobre-abandonado-delincuente" que conducen a galvanizar la subjetividad de los niños/as y/o adolescentes como menores y pobres abandonados y delincuentes que comparten una calificación estigmatizante como justificativo cultural y estatal, y el enmarcado de las subjetividades en la política preventiva criminal. 


\section{Crítica teórica de lo real II: la Convención de los Derechos de los Niños}

La CDN es ponderada en cuanto a la población que afecta "no solo hacia los niños y adolescentes carenciados sino [...] a todos ellos sin discriminación por sexo, situación económica, raza, religión, etc." (PROYECTO DE LEY $\mathrm{N}^{\mathrm{o}}$ 6354, p. 6). El enclave convencionalista pretende englobar las variadas características que pueden haber afectado a las niñeces, a diferencia de las legislaciones de la minoridad en riesgo donde solo el "menorabandonado" es el sujeto de la ley.

En cuanto a la subjetividad, se coloca en duda esta entidad en las legislaciones minoristas, ya que la niñez y adolescencia es administrada como objeto del derecho por medio de la tutela. Es así que el cambio fundamental introducido por la CDN es que los/as niños/as serán "conceptualmente" sujetos de derecho. Este cambio hace que deban "variar la aplicación de las políticas en la práctica” (PROYECTO DE LEY No 6354, p. 6). Sin embargo, el concepto no se desarrolla, aunque aseguran que impone un deber ser "variar la aplicación de las políticas".

La familia y la escuela, como instituciones de la sociedad, cumplen distintos roles. Cuando estos papeles se cumplen son primordiales para el "desarrollo humano integral" de los niños/as. Esta es "la premisa fundamental de la Convención" (PROYECTO DE LEY No 6354, p. 6). Sin embargo, expresan: "esta función no siempre se cumple en el total de la sociedad". Ante esta certeza, el Estado "debe brindar" el acceso "a todos" mediante instituciones que cumplan diferentes funciones a favor del desarrollo. Al decir, que el Estado garantiza a todos, desplaza la falta sospechada en los roles cumplidos por la familia y la escuela, hacia el total de la población. Es la introducción de un deber ser sobre un Estado propuesto por la Convención.

De esta manera, la traducción de la Convención (PILOTTI, 2001), es un interpretación local y legislativa aún en estado de concepto. Entienden que implicará una puesta en práctica de diversas políticas, motorizadas por un Estado particular. Es un Estado quimérico y global que pueda brindar accesos para los roles no cumplidos de la familia y la escuela.

¿Qué hace el nuevo proyecto?

Este nuevo proyecto de ley, se propone atender a la niñez en riesgo, pero también a la niñez en general, como la CDN. Esta niñez es entendida como sujeto de derechos, conceptualización que se afirma como un dogma, pero no se define en el texto argumental. Esta novedad de conceptos es propia de los inicios del discurso sobre derechos, donde se intentará demonizar y sacralizar un tipo de semántica en relación a la CDN (BARNA, 2012). 
Para la concreción de este objetivo se enumeran los cambios que detalla el nuevo proyecto. El primer cambio propuesto es la creación de dos juzgados diferenciados: Juzgado de Familia y en lo penal de menores. Este cambio ha sido celebrado por diferentes actores debido a la separación entre abandono y delito en la niñez y adolescencia (KONTERLLNIK; MUÑOZ; PELLIZA, 2002; FARIAS CARRACEDO, 2014). El segundo cambio propuesto es la especificidad tutelar como acoto a la tutela del juez sobre la niñez y adolescencia. Especificar la tutela de los jueces permite restringir la discrecionalidad judicial sobre la situación de la infancia. Para ello, la medida tutelar será decidida por el Juzgado de familia, no por Juzgado de Menores donde se dirimían causas penales y civiles. Este viraje administrativo de la medida, según la letra de los/as legisladores representa una división entre el lugar del niño y el adulto en el ámbito penal. Por otro lado, si bien no hay un cuestionamiento a la tutelaridad como potestad estatal para el control social de las infancias, la técnica propicia un cambio topográfico, implicando más que un cambio material sino una troca simbólica.

En cuanto a temáticas específicas de los circuitos judiciales: 1. se limita el procedimiento recursivo, 2. se establece etapa prejudicial, y, por último, 3 . le otorgan funciones específicas al Ministerio Publico, de tal manera que los mismos derechos, deberes y garantías de todo/a ciudadano/a se revaliden para la niñez en esta nueva función.

A su vez, surgen algunos reconocimientos relevantes propuestos por el proyecto de ley, aunque carecen de una metodología que especifique los modos de hacerse efectivo: se coloca a la sociedad en un lugar de garante y denunciante de los derechos de los niños que puedan violarse, velando por su dignidad; se reconoce el derecho de la familia al acto de crianza de sus hijos/as y se coloca en el lugar de excepción la crianza en hogares, y no debe ser privado de libertad sin debido proceso.

Por último, se plantea un reconocimiento que acota e intenta desarticular la judicialización de carencia material de las familias: "Carencia de recursos en familia no es motivo suficiente para quita de patria potestad. Debe ser asistido por programa asistenciales. La política de atención debe ser "encarada mediante un conjunto articulado" provincia, municipio y ONG" (PROYECTO DE LEY No 6354, p. 7). Aquí se propone, una función elemental para el Estado ejecutivo, frente a la pobreza de las familias. El Estado se propone como garante de los accesos a bienes y servicios que, mediante su falta, puedan colocar en sospecha la crianza de los/as niños/as en las familias.

Para propiciar la articulación institucional y evitar la segmentación sectorial se propone la creación del Consejo provincial del niño y adolescente. Este dispositivo está fuertemente 
cargado de expectativas y se le dedica un amplio espacio a su explicación. Esta extensión, no dada a los cambios judiciales, por ejemplo, refuerza la afirmación que el Consejo fue la primera opción antes de pensar en reformar el sistema completo, como explicitamos anteriormente.

Frente a la sectorialización propia de las distintas carteras políticas: educación, salud, deporte, etc., se propone la utopía de la articulación "que se hace imprescindible implementar a la luz de nuevas concepciones del niño como sujeto de derechos" (PROYECTO DE LEY N ${ }^{\circ}$ 6354, p. 7). Para ello, se propone el garantizar "el encuentro" entre los distintos sectores oficiales y los diferentes sectores.

Otro de los dispositivos que se crearán es la Dirección Provincial de niñez y adolescencia como "Espacio de gestión ejecutiva". En el ámbito municipal se propone una emulación a escala por medio de la creación de los departamentos de Niñez y Adolescencia, los que oficiarán de "caja de resonancia de los problemas de la niñez" cuya voz llevarán al consejo (PROYECTO DE LEY No 6354, p. 10).

Por último, la organización de las instituciones privadas, también tienen lugar en este proyecto, aunque su denominación es Organizaciones no gubernamentales. Expresa que tendrán representación en el Consejo para participar y es la Dirección quien las controlará: personería jurídica, autorizando y controlando el destino de fondos.

Estos son los fundamentos, que ofician que razones para la necesidad de cambiar lo existente. Después de la primera sanción en la cámara de Diputados, se envía a la Cámara de Senadores. En ese ámbito, se recibe una carta formal de la Suprema Corte de Justicia de la provincia de Mendoza que acompaña una nota con argumentos de la judicatura local. A continuación, presentamos el punto de vista de los magistrados.

\section{La carta de los magistrados}

El poder judicial, es decir la justicia de menores, estaba distribuida en cinco Tribunales de Menores en su mayoría en la primera circunscripción judicial con asiento en la ciudad capital de Mendoza. El 22 de diciembre de 1993, remitieron una nota con recomendaciones al texto aprobado en Cámara de Diputados.

Mientras la nota es recibida por los legisladores, a nivel nacional se estaba acordando la reforma de la Carta Magna Nacional. Fue la reforma más profunda desde la Constitución de 1853, y fue aprobada el 22 de agosto de 1994 (RAPOPORT, 2013). Entre las reformas realizadas, se otorga jerarquía institucional a la $\mathrm{CDN}$, junto a otros tratados internacionales (CN artículo 75 inciso 22). Es así que la carta de los magistrados tomó una relevancia diferente entre 
su emisión y la consideración legislativa de la modificatoria de la Carta Magna. La Convención podía presentarse como un nuevo horizonte frente a las leyes nacionales de infancias vigentes hasta el momento.

Los remitentes de la nota se identifican como magistrados de la Justicia de Menores de Mendoza. A su vez, ellos/as se encuentran "institucionalmente preocupados" por el tratamiento de la ley, porque representará "un cambio total al quehacer diario de nuestros tribunales". Más allá de que esta modificación significará un cambio para sus rutinas y cotidianeidades, ellos se interiorizarán en la propuesta por los "intereses supremos del niño" y porque están "a diario con la problemática del niño y adolescente” (PROYECTO DE LEY No 6354, p. 179). Esta autopercepción, nos hace preguntarnos: si sólo ellos, los magistrados, están en contacto cotidiano con los problemas de la infancia, ¿qué sucede con los agentes estatales de dispositivos provinciales o municipales de diferentes sectores? ¿También quedarían por afuera los voluntarios de las ONG que atienden en distintos espacios de la sociedad? Esta definición dada por los magistrados genera una división entre un "ellos" y un "nosotros" que se expresa en la crítica hacia los legisladores al cambiar esta ley legendaria. La frase que sustenta la crítica hacia este proyecto de ley es: "un avance del órgano administrador (Directorio) sobre facultades propias de la judicatura” (PROYECTO DE LEY No 6354, p. 179).

La posición judicial y su pragmatismo

En la carta de los magistrados se plantean críticas absolutas, donde solicitan derogar artículos y secciones completas, pero también se plantean adaptaciones a las sugerencias de la ley que denotan la astucia para fortalecer su posición como aparato y tecnología judicial.

En primer lugar, plantean que el proyecto de ley "triplicaría la tarea de los actuales Tribunales de Menores" (PROYECTO DE LEY No 6354, p. 180) y agregan: "Estimamos que en total deberá proveerse la existencia de aproximadamente 15 juzgados, de los cuales deberá determinarse en forma clara la competencia de cada uno" (PROYECTO DE LEY No 6354, p. 181). Fundamentan esta posición dado que existe un conflicto de competencias al anexar funciones que pertenecen a las Defensorías de Menores e Incapaces y a los Juzgados Civiles, a las tareas tradicionales de los Juzgados de Menores.

Suman a sus sugerencias, que para esta propuesta de especialización del aparato judicial se propone el cambio de nomenclatura de los juzgados, de juzgado de menores a juzgado de familia, y la creación de los restantes a fin de llenar ese cupo establecido. Claramente se propone crear 10 juzgados que son los que restan para los 15 juzgados solicitados. Es más, resaltan en mayúsculas que "resulta indispensable incluir [su pedido] ${ }^{14}$ en la letra de la ley" 
(PROYECTO DE LEY Nº 6354, p. 181). Este reaseguro solicitado, aumentaría el presupuesto para el poder judicial y, si estuviere en la ley, existiría la posibilidad de exigirlo mediante diferentes recursos judiciales.

Proponen, a su vez, que el juzgado de menores se llame Tribunal de Protección y Penal y atienda las cuestiones sobre la minoridad. Para completar su plano arquitectónico realizan la correcta articulación de competencias entre el juzgado de familia y el Juzgado de Menores.

En los fundamentos (título IV) se establece que la falta de división entre lo penal y lo civil, ha contribuido construir el complejo entramado menor-delincuente-abandonado y pobredelincuente-abandonado. En la misiva judicial disienten con este punto de vista debido a: 1.Sobre las competencias: "Resultará imposible en la práctica delimitar la competencia de los Tribunales como lo pretende la nueva Ley"; 2.- Sobre el niño en tutela del juez: "No cabe la posibilidad de que un niño que está siendo tutelado por un juez, por una situación familiar de larga data conocida, deba ser derivado a otro juez por razones de competencia por materia"; 3.Sobre la celeridad y simpleza en la actuación: "Todo ello destruye el principio de celeridad y simpleza que debe primar en estas actuaciones" (PROYECTO DE LEY Nº354, p. 181).

Existe una fuerte crítica a los dispositivos creados por la ley, ya que los caracterizan como burocráticos, cuando "en materia de minoridad debe primar la celeridad y la inmediatez". Esta crítica se realiza a dos dispositivos "el procedimiento prejudicial y de avenimiento" anterior al procedimiento ordinario y al registro centralizado de pretensos adoptantes. Solicitan la derogación de estos dispositivos ya que la garantía de todo procedimiento siempre ha sido el Juzgado y no el organismo administrativo, por lo que, si se concretarán estos dispositivos, estarían lejos del "ojo tutelar" del juez.

Por último, entiende que sus funciones son las derivadas por el Patronato, y que debe el artículo que expresa lo siguiente:

Artículo $\mathrm{N}^{\circ} 7 \mathrm{La}$ falta o carencia de recursos materiales de los padres, tutor o guardador, no constituye causa suficiente para la exclusión del niño o del adolescente de su grupo familiar, si no existiera otro motivo grave que autorizara por sí mismo la imposición de la medida. Asimismo, será de interpretación restrictiva a los efectos de decidir sobre la suspensión o pérdida de la patria potestad, en cuyo caso el grupo familiar deberá ser incluido en programas de asistencias (PROYECTO DE LEY N ${ }^{\circ}$ 6354, p. 182).

Deben derogarse "totalmente", en base a las siguientes razones: pretende limitar las facultades jurisdiccionales propias de la magistratura; es una excepción que carece de asidero; el Juez está facultado para adoptar amplias medidas tutelares cuando un menor aparece en 
situación de desamparo moral o material; Decir que por falta de medios económicos de los padres o carencias no se pueden adoptar medidas tutelares resulta una incongruencia.

Apoyan su opinión sobre la ley en base a las siguientes normativas jurídicas: "los arts. 6; art. 9; Art. 20 inc.; Art. 24 inc. a, b, y c; art. 39 in fine de la Declaración de los Derechos del Niño” (PROYECTO DE LEY Nº 6354, p. 182). Más allá del articulado que sospechosamente en la CDN se sustentaría en la tutela dirigida por los jueces, incurren en el error de confundir a la Convención con una Declaración. Sin embargo, la diferencia entre Declaración y Convención son sustanciales, ya que la Convención (1989) obliga a los Estados a tomar en su parte en sus gobiernos de los postulados de la misma, mientras que la Declaración (1959) no tiene carácter obligatorio para los Estados, porque no son documentos jurídicamente vinculantes.

Después de la carta de los magistrados

Entre lo propuesto por los magistrados y la resolución de los/as legisladores, muchas de las recomendaciones no fueron tenidas en cuenta. Esto hace suponer que el tiempo de las sugerencias era anterior a la incorporación en la Constitución Nacional de los tratados de Derechos Humanos a partir de la modificatoria del año 1994, como mencionamos anteriormente.

Desde la presentación de la carta el día 22 de diciembre de 1993, a la nueva convocatoria presencial de los magistrados para el día 17 de agosto de 1995, pasaron 1 año y 9 meses. No podemos asegurar si se presentó el nuevo proyecto o se profundizó en los puntos de la carta. Aunque descubrimos un texto suelto en el cuerpo del expediente que plantea lo siguiente:

La presente ley entrará en vigencia a partir de promulgación, con excepción de lo dispuesto para la organización de la justicia de Familia y en lo Penal de Menores y sus procedimientos hasta tanto se cumplimente lo normado por los art. 204 y 205 de esta ley (PROYECTO DE LEY No 6354, p. 228).

Esta condicionalidad, ya la vimos expresada en la carta de los magistrados, cuando solicitaban la ampliación del aparato judicial y queda explícitamente designados en los artículos de la Ley 6354 art. 204-205 que solicitan previo cumplimiento a la promulgación.

De esta manera, la posición judicial detenta el monopolio de la minoridad (mención utilizada por ellos/as) amparados en su derecho de ejercicio del Patronato y en la relectura conveniente del instrumento internacional que, debido a la juventud de su aprobación, no cuenta con la significación y traducción interpretativa de la aplicación en los territorios. A su vez, y tal como lo afirma Grinberg (2013) funcionan como "espíritu de cuerpo", es decir corporativamente, buscando resistir la pérdida de su histórica exclusividad la administración de la infancia pobre. Asimismo, lo que evidencia este estudio, es la modulación temporal de la 
resistencia, al plantear su pragmatismo en la concesión y negociación de las propuestas del proyecto de ley.

\section{Consideraciones finales}

Este estudio tuvo como objetivo analizar el proceso de producción de la primera ley argentina de adecuación a la CDN a través de la caracterización de la autora-legisladora, los fundamentos de la normativa propuesta y la respuesta del actor judicial, en la primera mitad de la década del 90.

En primer lugar, destacamos que la autora de la ley, una de las primeras mujeres en ingresar a la legislatura después del retorno democrático, también fue Interventora de la Dirección Provincial del Menor, Diputada y Senadora de la provincia de Mendoza. Asimismo, la sustanciación de su accionar surgía por su militancia política y social y la participación en espacios bisagra donde legisló aquello que primero gestionó. Sumado a ello, su labor parlamentaria fue basta, frente a una estructura política donde primaba la matriz patriarcal y machista, que le acotaba las propuestas según las expectativas de la época (mujer, niñez y cuidados).

Los fundamentos o razones presentados por la legisladora y los coautores, sugerían los primeros frentes de batalla en razón del discurso dicotómico y legitimado sobre los paradigmas de la protección integral. Este discurso se caracteriza como un frente teórico que critica la realidad vigente del Patronato local y nacional, denominada Doctrina de la Situación Irregular. Aún no se plantea la protección integral como un paradigma, sino que se sustentan los cambios en los postulados de la CDN.

Resulta interesante observar como las ideologías imperantes en la época se centran, por un lado, en la crítica al etiquetamiento y estigmatización social como el flagelo de las infancias y adolescencias, y por el otro, en la pobreza en perspectiva criminológica de subcultura, lo que dificulta la visión del delito en los sectores medios y altos. No podemos dejar de rescatar la mirada crítica hacia el estado benefactor y asistencialista, en los albores de la llegada neoliberal, que propone un Estado mínimo y habilita acciones del mercado en desmedro de lo anterior. Esta perspectiva sirvió como base para la llegada de la acción neoliberal en la región.

En cuanto a la tutela, acordamos con aquellos estudios (KONTERLLNIK; MUÑOZ; PELLIZA, 2002, OCAÑA; LAVADO, 2008, FARIAS CARRACEDO, 2014; MOLINA de JUAN, 2017) que plantean a la primera ley de infancia a nivel nacional como pionera, aun cuando la competencia tutelar todavía es ejercida por el juez. Sin embargo, es preciso aclarar 
que el cambio en la tutela se plantea en una doble vía: frente al poder con el que se enfrentó y a la modificación que introdujo. Esta modificación se dio a partir de la introducción de división de competencia civil-penal a través del nuevo aparato judicial, prohibición de tomar medidas solo por razones de carencia material, al encumbrarse al órgano administrador como institución propositiva cuando era como subsidiario exclusivo del poder judicial y, por último, al presentar a la CDN en su capacidad de acción, cuando se denominaba por la magistratura como Declaración.

En cuanto al poder judicial, plantea sus resistencias sin rodeos a través de la carta enviada al poder legislativo en la que esbozan que es un avance del órgano administrador sobre el poder judicial. Critican algunas de las nuevas ideas como burocráticas, cuando en realidad estos temas requieren acciones diligentes y simples tomadas por un juez. Se autoperciben como el ojo tutelar que conoce la problemática de la niñez y adolescencia, ya que trabajan cotidianamente con ella. Por último, plantean que las nuevas tareas triplicarían las tareas actuales y proponen ampliar el aparato judicial sustancialmente. Esta propuesta logró el reaseguro mediante el articulado de la ley sancionada. Esta maniobra denota el pragmatismo judicial y la sagacidad de sus propuestas en la reproducción del propio funcionamiento.

Para futuras investigaciones y posteriores estudios, sería interesante indagar vinculación o articulación entre los/as aliados/as y adversarios/as del proceso de implementación del enfoque de derechos, tanto en el proceso legislativo como en la posterior sanción de la Ley Provincial $\mathrm{N}^{\circ}$ 6354. Otras de las indagaciones pendientes surgen de la convergencia entre fuentes, para evaluar el alcance los pedidos de la magistratura y posterior sanción de la ley 6354.

Para finalizar, entendemos que la implicancia de estudio permite conocer a los actores en el ejercicio del poder después de la ratificación de CDN. A pesar de que estas resistencias persistieron, este primer enfrentamiento contribuyó a reorganizar y redefinir acciones y representaciones sobre los modos y maneras de la burocracia institucional sobre los derechos de las infancias a partir de la década del 90.

\section{Referencias bibliográficas}

ALVARADO, Mariana; BARÓN DEL PÓPOLO, Guillermo. "Nosotras sembramos al voleo". Una conversación con Sofía D'Andrea sobre el Tercer Encuentro Nacional de Mujeres (Mendoza, 1988) y el Grupo Ecuménico de Mujeres. Boletín GEC: Teorías Literarias y prácticas críticas, Mendoza: Universidad Nacional de Cuyo, n.23, p.159-183, 2019. 
BARNA, Agustín. Convención Internacional de los Derechos del Niño: hacia un abordaje desacralizador. Kairos: Revista de Temas Sociales, San Luis: Universidad Nacional de San Luis, n. 29, p. 1-19, 2012.

BELOFF, Mary. Modelo de la protección integral de los derechos del niño y de la situación irregular: un modelo para armar y otro para desarmar. Justicia y Derechos del Niño, Montevideo: Instituto Interamericano del Niño, la Niña y Adolescentes, v.01, p. 9-21, 1999.

BOURDIEU, Pierre. La force du droit. Actes de la Recherche en Sciences Sociales, Paris: Persée, v.64, p. 3-19, 1986.

BRACHETTA, María Teresa; BRAGONI, Beatriz; MELLADO, Virginia; PELAGATTI, Oriana. Te contamos una historia de Mendoza (de la conquista a nuestros días). Mendoza: Ediunc, 2019. 218p.

CAMINOTTI, Mariana. Derribar los muros indebidos: Reflexiones en torno de las leyes de cupo femenino en argentina. Revista Aportes para el Estado y la Administración Gubernamental, Buenos Aires: Asociación de Administradores Gubernamentales, v. 14, n.25, p. 13-33, 2008.

CARBONETTI, Adrián. Historia de una epidemia olvidada. La pandemia de gripe española en la argentina, 1918-1919. Desacatos, Ciudad de México: Centro de Investigaciones y Estudios Superiores en Antropología Social, n.32, 2010, p. 159-174.

CASAS, Ferrán. Infancia y representaciones sociales. Política y sociedad, Madrid: Universidad Complutense de Madrid, v.43 n.01, p. 27-42, 2006.

CASTELL, Robert. De la peligrosidad al riesgo. En: WRIGHT MILLS, Charles; FOUCAULT, Michel; POLLAK, Michael; MARCUSE, Herbert; HABERMAS, Jurgen; ELIAS, Norbert; BOURDIEU, Pierre; GOFFMAN, Erving; BERSTEIN, Basil; CASTELL, Robert (Eds.). Materiales de sociología crítica. Madrid: Ediciones de La Piqueta, 1986. p. 219-243.

CERDÁ, Juan Manuel. Una política social particular para la infancia en década del '30: patronato y tribunales de menores en la provincia de Mendoza. Revisa Digital de la Escuela de Historia, Rosario: Universidad Nacional de Rosario, v.05, n.08, 2013, p. 196-220.

CONSEJO FEDERAL DE DESARROLLO SOCIAL; SECRETARÍA DE DESARROLLO SOCIAL DE LA PRESIDENCIA DE LA NACIÓN; ASOCIACIÓN DE MAGISTRADOS Y FUNCIONARIOS DE LA JUSTICIA DE MENORES; SECRETARIADO NACIONAL PARA LA FAMILIA DE LA CONFERENCIA EPISCOPAL ARGENTINA; UNICEF. Análisis de las conclusiones. En: ENCUENTRO FEDERAL DE POLÍTICAS SOBRE INFANCIAS Y ADOLESCENCIAS 1998. Mendoza. 1998. 54 p.

DIRECCIÓN DE ESTADÍSTICAS E INVESTIGACIONES ECONÓMICAS (DEIE). Índice de Privaciones Múltiples en los niños, niñas y adolescentes. 2020. Disponible en: https://bit.ly/2Z0xGGq. Consultado en: 14.11.2021.

FARIAS CARRACEDO, Ana Carolina. Legislación acorde a La doctrina de La protección integral: Mendoza, província pionera. Niños, menores e infancias, La Plata: Universidad Nacional de La Plata, n. 08, p.1-7, 2014.

GARCÍA MÉNDEZ, Emilio Derecho de la infancia-adolescencia en América Latina: de la Situación Irregular a la Protección Integral. Bogotá: Forum Paces, 1994. 455p. 
GOBIERNO DE MENDOZA. Ley provincial $N^{o} 1304$ Patronato y Tribunales de Menores. 1939.

GOBIERNO DE MENDOZA. Ley provincial $N^{o} 6354$ Régimen Jurídico de protección de la minoridad. 1995.

GOBIERNO DE MENDOZA. Poder Legislativo. Proyecto de ley $N^{o}$ 6354. 1993.

GOMES DA COSTA, Antônio Carlos. De menor a cidadão: notas para uma história do novo direito da infância e da juventude no Brasil. Brasília: Ministério de Ação Social. 1993. 72p.

GRINBERG, Julieta. La recepción de "los derechos del niño" en argentina: trayectorias de activistas y conformación de una nueva causa en torno a la infancia. Virajes, Caldas: Universidad de Caldas, v.15, n.01, p.299-325, 2013.

KONTERLLNIK, Irene; MUÑOZ, Teresa; PELLIZA, Cecilia. Protección de Derechos de la Niñez a nivel local en Argentina. El caso Guaymallén: un modelo en construcción. En FERRARI, Mario; COUSO, Jaime; CILLERO, Miguel; CANTWEL, Nigel (Eds.). Internación de Niños, ¿El comienzo del fin? Crisis de los internados y transformación de las políticas de infancia en España, Italia y el Cono Sur. Santiago de Chile: Productora Gráfica Andros, 2002. p.73-84.

LLOBET, Valeria. La producción de la categoría "niño-sujeto-de-derechos" y el discurso psi en las políticas sociales en argentina. Una reflexión sobre el proceso de transición institucional. En: LLOBET, Valeria (Org.) Pensar la Infancia desde América Latina: Un estado de la cuestión. Ciudad Autónoma de Buenos Aires: CLACSO, 2013.p. 209-235.

LUCERO, María Victoria. Infancia y derechos: radiografía crítica de la Ley 26.061: escenarios provinciales normativos, institucionales y jurisprudenciales. Buenos Aires: EUDEBA, 2013. 424p.

MOLINA de JUAN, Mariel. El sistema de protección integral de derechos de niñas, niños y adolescentes en la provincia de Mendoza. En: FERNÁNDEZ, Silvia Eugenia (Ed.). Tratado de derechos de niños, niñas y adolescentes. Tomo II. Buenos Aires: Abeledo Perrot, 2017. p. 18231844.

OCAÑA, Viviana Estela. La infancia judicializada. En ROIG, Arturo; SATLARI, María (Ed.). Mendoza, identidad, educación y ciencias, Mendoza: Ediciones Culturales de Mendoza, 2007. p.350-400.

OCAÑA, Viviana Estela; LAVADO, Diego. Comentarios sobre la ley de protección integral del Niño y el Adolescente de la provincia de Mendoza. En: BELOFF, Mary (Ed.). La protección a la infancia como derecho público provincial, p.383-422. Buenos Aires: Adhoc, 2008.

OLDRÁ DE BERCHESSI, M. T. Análisis de dos años de gestión. Mendoza. 1990. Documento inédito.

ORGANIZACIÓN DE LAS NACIONES UNIDAS. Convención de los Derechos del Niño. 20 de noviembre de 1989.

PETRY VERONESE, Josiane. Direito da criança e do adolescente. Rio de Janeiro: Lumen Juris. 2017. 367p.

PILOTTI, Francisco. Globalización y Convención sobre los Derechos del Niño: el contexto del texto. Santiago de Chile: CEPAL, 2001. 84p. 
Fronteiras - Revista Catarinense de História | https://periodicos.uffs.edu.br/index.php/FRCH/index

ISSN 2238-9717 | n. 38, p. 78-101, jul.-dez./2021 | DOI: https://doi.org/10.29327/253484.1.38-6

PINEDA NAVAS, Armando. La Técnica Legislativa en Centroamérica y República Dominicana. San José: IIDH, 2001. 203p.

PODER LEGISLATIVO DE LA PROVINCIA DE TIERRA DEL FUEGO. Diario de sesiones $N^{o} 19.1997$.

RAPOPORT, Mario. Historia económica, política y social de la Argentina (1880-2003). Buenos Aires: Emecé, 2013. 1037p.

SALINAS, Pablo. Criminología Aplicada. Mendoza: Universidad del Aconcagua. 2020. 143p.

ZAPIOLA, María Carolina (2010). La ley de Patronato de Menores 1918 ¿Una bisagra histórica? En: LIONETTI, Lucía; MÍGUEZ, Daniel (Org.). Las infancias en la historia argentina. Intersecciones entre prácticas, discursos e instituciones (1890-1960). Rosario: Prohistoria, 2010. p. 117-132.

\section{Agradecimiento}

El presente trabajo fue realizado en el marco de la Beca Interna Doctoral de CONICET del primer autor.

Los autores agradecen a las editoras y a los revisores anónimos por sus valiosos aportes y comentarios.

Recebido em 02/10/2021. Aceito em 08/11/2021.
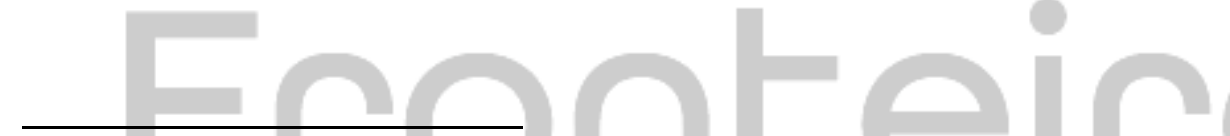

${ }^{1}$ Mendoza es una provincia de Argentina ubicada en la zona centro-oeste del país. Actualmente, cuenta con una
población de 1.736 .929 habitantes, de los cuales se estima que el 42,1\% se encuentra en situaciones de pobreza e
indigencia. Para el año 2019 , el $50 \%$ de los niños, niñas y adolescentes de la provincia se encontraba en situación
de privación de alguno de sus derechos fundamentales de salud, educación, vivienda, saneamiento, protección social y/o acceso a la información (DEIE, 2020).

${ }^{2}$ A lo largo del texto se coloca en negrita aquello que ha sido destacado por los autores de las fuentes consultadas.

${ }^{3}$ Dichos de realizados con frecuencia por una de las senadoras que conformaba la cámara Sra. Marta Stagni, que invitaban a estar siempre en la vanguardia como estrategia política y de supervivencia.

${ }^{4}$ Se presentó como Diputada por la Tercera Sección Electoral desde 1983-1989 por el Partido Justicialista. En el año 1993 se presentó y fue electa como Senadora por el Partido Justicialista, en la misma sección.

${ }^{5}$ Se presentó por la Cuarta Sección Electoral por la Alianza de Centro, según figura en Acta de proclamación de candidatos electos en los comicios elecciones el 14 de mayo de 1989.

${ }^{6}$ Se presentó por la Segunda Sección Electoral por la UCR (Unión Cívica Radical) según figura en Acta de proclamación de candidatos electos en los comicios el 14 de mayo de 1989.

${ }^{7}$ La historia del macrohogar en Mendoza puede indagarse en FARIAS CARRACEDO, Carolina. Historia del Hogar Casa Cuna de la ciudad de Mendoza: la institución que permanece. INFEIES. Revista Científica Multimedia sobre la Infancia y sus Institucion(es), v. 4, n. 4, p. 64-96.,2015.

${ }^{8}$ Según Caminotti (2008): “el predominio masculino es un rasgo tan constitutivo y persistente en la vida política que, en 2007, el $83 \%$ de los parlamentarios nacionales del mundo eran varones y la participación de mujeres en los más altos cargos ejecutivos resultaba ciertamente exigua". Es por ello, que la ley de cupos estaba "ideadas para enfrentar las desventajas que han mantenido a las mujeres al margen del acceso a cargos electivos" (p. 14).

${ }^{9}$ Estas reticencias pueden observarse con claridad en la entrevista realizada por Alvarado y Barón Del Pópolo (2019) sobre el Tercer Encuentro Nacional de Mujeres organizado en Mendoza en el año 1988, Sofía D'Andrea (militante feminista y por los derechos humanos, peronista revolucionaria, docente y periodista) expresa que el gobernador Bordón irrumpió en el encuentro, donde las participantes "no querían que participara", realizando el anuncio de la creación de una Asesoría de la Mujer. Si bien fue un anunció esperado, comentaba que después dijo comentarios descriptos como "raros" y "extraños" por la entrevistada, demarcando un clima de época donde se 
afirma la subsidiaridad de la mujer al precepto masculino y las advertencias sobre las reuniones entre mujeres. La entrevistada lo expresa de la siguiente manera: "dijo cosas raras, cosas como que "porque hay que compartir con los varones..." Esto de "ojo de juntarse solas ustedes". ¿Viste cuando te tiran flores? "Las mujeres tienen fuerza, iniciativa, pero sin nosotros... ¿qué van a hacer?” (p.162).

${ }^{10} \mathrm{El}$ proyecto se presentó en la cámara de diputados bajo № 14.410 y se le otorgó media sanción el 24/11/1993. En cámara de senadores, se retomó con el N²2.083/93, se le realizaron modificaciones y se envió en revisión a la cámara de Diputados, donde se sancionó positivamente incluyendo las modificaciones realizadas el 22/11/1995. Se promulgó como la ley provincial $\mathrm{N}^{\circ} 6354$ Estableciendo la protección del niño y el adolescente.

${ }^{11}$ Este vínculo será indagado en los próximos estudios con mayor énfasis, ya que, si bien no podemos precisar la modulación de esta vinculación, tenemos la certeza de esta articulación entre la legisladora y el consultor de UNICEF, en esta parte del proceso.

${ }^{12}$ En Argentina la criminología positivista comienza a difundirse a finales del siglo XIX y principios del siglo XX. Se basaba en las señales antropológicas de la criminalidad. De esta manera Ingenieros: "funda el instituto de Criminología Nacional en 1907 con archivos de antropología criminal y dio clases en la Universidad de Lausana, Suiza. Ingenieros trabaja clasificando a los presos y adhiere a las tesis psicologistas" (Salinas, 2020, p. 34).

${ }^{13}$ No tenemos la seguridad si en realidad era una creencia local que todos por igual son perversos o en realidad quisieron colocar peligrosos.

${ }^{14}$ Lo introducido entre corchetes es nuestro, para facilitar la comprensión de lo planteado en la fuente. 